\title{
Sustentabilidad y prácticas institucionales en los hábitos sostenibles de estudiantes universitarios de Puerto Rico'
}

\author{
Silvia López Paláu', Beatriz Rivera Cruz²
}

Resumen: A partir de los años 90s se reclama que las instituciones de educación superior promuevan la sostenibilidad. En Puerto Rico hay una necesidad imperiosa de investigaciones conducentes a facilitar la comprensión de la educación de y para la sostenibilidad y el rol de las instituciones de educación superior en su contexto local. Este estudio utiliza como marco teórico el modelo transteórico de etapas de cambio de Prochaska \& Di Clemente (1984) y las aportaciones de Mejía y Serna (2015) y Gudynas (2004, 2009, 2011 ), que describen los fundamentos de la sustentabilidad débil, fuerte y superfuerte, y la definición de Campus Sostenible de Castañeda \& Quintero (2015). Los resultados revelan que el proceso de adopción de comportamientos sostenibles puede ser positivamente promovido por las prácticas institucionales.

Palabras clave: sustentabilidad, campus sostenible, modelo transteórico de etapas de cambio, sostenibilidad, educación superior.

Fecha de recepción: 17 de marzo de 202I.

Fecha de admisión definitiva: 3I de diciembre de 2021.

${ }^{1}$ Artículo presentado en el $6^{\circ}$ Congreso Internacional de Investigación Educativa USAL-PALECH "CLIE $2021^{\prime \prime}$

${ }^{2}$ Universidad de Puerto Rico. 
Sustainability and Institutional Practices in the Sustainable Habits of University Students in Puerto Rico

\begin{abstract}
Since the 90s higher education institutions are called to promote sustainability. In Puerto Rico there is an urgent need for research leading to facilitating the understanding of education for sustainability and the role of higher education institutions in their local context. This study uses as a theoretical framework the transtheoretical model of stages of change of Prochaska \& Di Clemente (1984) and the contributions of Mejía and Serna (2015) and Gudynas (2004, 2009, 2011) that describe the fundamentals of weak, strong and super-strong sustainability orientation, and the sustainable campus definition by Castañeda \& Quintero Campus (2015). The results reveal that the process of adopting sustainable behaviors can be positively promoted by institutional practices.
\end{abstract}

Key words: sustainability, sustainable campus, transtheoretical model of stages of change, sustainability orientation, higher education

\section{La durabilité et les pratiques institutionnelles dans les habitudes durables des étudiants de l'université de Puerto Rico}

Résumé: Depuis les années 1990, des appels ontété lancés aux établissements d'enseignement supérieur pour qu'ils encouragent la durabilité. À Porto Rico, il existe un besoin urgent de recherche pour faciliter la compréhension de l'éducation à la durabilité et du rôle des établissements d'enseignement supérieur dans leur contexte local. Cette étude utilise comme cadre théorique le modèle transthéorique des étapes du changement de Prochaska \& Di Clemente (1984) et les contributions de Mejía et Serna (2015) et de Gudynas (2004, 2009, 201 1), qui décrivent les fondements de la durabilité faible, forte et super-forte, ainsi que la définition de Campus durable de Castañeda \& Quintero (2015). Les résultats révèlent que le processus d'adoption de comportements durables peut être favorisé par les pratiques institutionnelles.

Mots clé: durabilité, campus durable, modèle transthéorique des étapes du changement, enseignement supérieur.

\section{Introducción}

A partir de los años 90 s el desarrollo sostenible se torna en uno de los principales objetivos de política pública en el mundo. El desarrollo sostenible (muy utilizado como sinónimo de sostenibilidad o sustentabilidad) se define en el Informe Brundtland (1987) como aquellos caminos de progreso social, económico, político y ambiental que satisfacen las necesidades del presente sin comprometer la capacidad de las futuras generaciones para satisfacer sus propias necesidades. Esa definición fue calificada como ambigua por muchos sectores. El debate, hoy en día, está dividido en tres grandes tendencias: débil, fuerte, superfuerte (Mejía y Serna, 2015; Gudynas, 2004, 2009, 2011). La sustentabilidad débil, con una fuerte perspectiva economicista; la sustentabilidad fuerte reclamando un cambio de paradigma que corrija al modelo económico (Pérez, Rojas y Ordóñez, 2016), 
y la sustentabilidad superfuerte, que plantea cambios profundos en los procesos productivos y la redefinición de diversos conceptos tradicionales.

Las instituciones de educación superior son uno de los actores responsables de la formación de los profesionales que habrán de responder a los retos de la sostenibilidad (Piza y otros; Sandri y otros, 2018). Cortés (2014) señala que estas instituciones están llamadas a ofrecer programas académicos que promuevan cambios sociales y progreso; a realizar investigaciones conducentes a encontrar soluciones a los problemas de la sociedad; a producir conocimiento sensible sobre los problemas ecológicos y sociales globales; y a preparar a los estudiantes en las competencias de la vida política y ciudadana, entre otras. Cada vez más voces requieren acciones educativas que transformen concepciones, hábitos y perspectivas para avanzar colectivamente hacia una sociedad sostenible. La educación superior es una herramienta crucial para la sostenibilidad que además de permitir que las personas adquieran y generen conocimientos, también debe propiciar que reflexionen sobre los efectos y la complejidad del comportamiento y decisiones desde una perspectiva de responsabilidad orientada hacia el futuro. El objetivo final es extender los cambios de actitud y comportamiento al conjunto de actividades que realizamos como consumidores, profesionales y ciudadanos (Vilches y Gil 2012; Vilches y otros, 2020).

Las instituciones educativas, y particularmente aquellas de las ciencias económicas y empresariales, tienen el reto de formar a los futuros directivos y directivas para que sean agentes de cambio, y contribuyan a alcanzar todos los objetivos de desarrollo sostenible definidos a nivel internacional, contribuyendo a sociedades más justas, sostenibles y solidarias, con instituciones más eficaces, responsables e inclusivas (Alcañiz y otros 2017). En los últimos años se ha abordado el concepto de Campus Sostenible como una estrategia de gestión a esos efectos. De esta forma se combina el aprendizaje formal y el informal dentro de las instituciones de educación superior lo cual, Barth y otros (2007) encontraron pertinentes para desarrollar competencias para la sustentabilidad. En resumen, es imprescindible que las instituciones de educación superior incorporen en todas sus actividades las múltiples y complejas facetas de la sostenibilidad (Labanauskis, 2017).

Explorar la orientación de los estudiantes hacia la sustentabilidad, así como las prácticas sustentables en su campus es crucial para el futuro de muchas universidades públicas que enfrentan una reducción en el financiamiento de sus operaciones amenazando su supervivencia. Tal amenaza afecta la movilidad social de los ciudadanos que de ella se sirven poniendo así en peligro la recuperación económica y social del contexto económico en el que enclava. En Puerto Rico la 
discusión teórica de la sustentabilidad, en general, ha ido ganando la atención de la academia en años recientes (Soto, 2012; Vila 2016; Vilela, 2014, Estrada y Olivares, 2017), pero no así aplicándola al contexto de la educación superior, ni en la producción de estudios empíricos, los cuales son muy escasos (López Paláu y Rivera Cruz, 2018, 2020; Rivera Cruz y López Paláu, 2017). Hay una necesidad imperiosa de investigaciones conducentes a facilitar la comprensión de la educación de y para la sostenibilidad y el rol de las instituciones de educación superior en su contexto local.

\section{I.I. Objetivos}

Este estudio pretende contribuir a llenar ese vacío en la literatura examinando el efecto de la percepción sobre las prácticas institucionales y la orientación hacia la sustentabilidad en los hábitos sostenibles de estudiantes de administración de empresas de Puerto Rico. En esa dirección los objetivos específicos son los siguientes:

1. Determinar la orientación de los estudiantes hacia el enfoque de sustentabilidad débil, fuerte o super fuerte $\circ$ insostenibilidad mediante el cálculo de cuatro índices.

2. Identificar la práctica actual de los estudiantes de comportamientos individuales sostenibles, mediante el cálculo del Índice de Prácticas Individuales Sostenibles.

3. Identificar prácticas institucionales congruentes con el concepto de campus sostenible y la percepción de los estudiantes sobre su práctica actual, mediante el cálculo del índice de Campus Sostenible.

4. Determinar el efecto de la orientación hacia la sostenibilidad y la percepción de campus sostenible en los hábitos sostenibles de los estudiantes.

\section{Revisión de literatura}

\section{I. Modelo transteórico de etapas de cambio}

Este modelo fue concebido inicialmente en Prochaska \& Di Clemente (1983) para ayudar a profesionales de la salud a entender como los fumadores pueden dejar de fumar y en Prochaska \& Di Clemente (1984) proponen el modelo transteórico formalmente. El modelo pretende explicar la intención de cambio de comportamiento 
proponiendo que dichos cambios ocurren en una serie de fases incrementales y no en un solo paso. Propone cinco etapas de cambio: pre-contemplación, contemplación, preparación, acción y mantenimiento. Estas etapas han sido validadas para explicar el cambio de diversos comportamientos (Prochaska et al., 1992, 1993, 1994, 2005; Lipschitz et al., 2005, Hally Rossi, 2008; Migneault et. al., 1999; Miller and Rollnick, 2002; Rossi et.al., 2000; Frestone y Mcgoldrick; 2008; López Paláu y Rivera Cruz, 2018; Rivera Cruz y López Paláu, 2017) ganando una amplia aceptación en los campos de la psicología, la psiquiatría y otros.

Frestone and McGoldrick (2008) adaptaron el modelo para examinar las motivaciones de los individuos para comportarse como consumidores responsables. Aesos efectos definen la etapa de pre-contemplación como aquella en la que los individuos no le dan mucho pensamiento al asunto ético particular y es muy probable que no esté inclinado a tomar ninguna acción. En la fase de contemplación el individuo está más consciente del asunto en cuestión y comienza a mostrar algunos signos de inquietud y se abre a nueva información. En la etapa de preparación la persona comienza a motivarse y su actitud hacia el cambio se va haciendo más positiva. Se va convenciendo de que algo hay que hacer. En la etapa de acción la persona cambia el comportamiento ejecutando pequeñas o grandes acciones. En la fase de mantenimiento el individuo sigue creyendo que vale la pena mantener la acción respecto al asunto ético. Lopez Paláu y Rivera Cruz (2019) encontraron evidencia de apoyo para sustentar la integración del modelo transteórico de las etapas de cambio de Prochaska \& Di Clemente (1984) a un modelo de toma de decisiones éticas. Los resultados aportaron evidencia de que los cambios de comportamientos no ocurren en un solo acto, sino que es un proceso de varias fases.

\subsection{Sustentabilidad}

El debate sobre el tema de la sustentabilidad está dividido en tres grandes tendencias: débil, fuerte, superfuerte. Las diferencias entre una postura y otra implica la ampliación y profundización de diversos elementos en los fundamentos de cada una. La sustentabilidad débil que se observa al amparo de conceptos de la económica neoclásica. Esta propuesta defiende que se debe sustentar el capital agregado de la sociedad (capital hecho por las personas y el capital natural) de forma tal que posibilite un nivel de consumo no decreciente en el tiempo, partiendo del principio de que el bienestar humano depende de la utilidad derivada de ese consumo y dando por sentado de que ambos capitales son sustituibles. La debilidad de esta tendencia se debe a la suposición de que capital natural puede ser compensado o sustituido por otras formas de capital o por el progreso tecnológico evadiendo 
así el problema de su agotamiento (Cabeza, 1996; Martínez-Alier y Roca, 2013; Víctor, 1991). Esta tendencia se caracteriza por una fuerte perspectiva economicista de los temas ambientales descansando en los juicios y decisiones de los técnicos.

La sustentabilidad fuerte que avanza en la crítica a la ideología del progreso o crecimiento. (Constanza, 1991; Constanza y Pattern, 1995; Daly, 1989; Howarth y Norgaard, 1993, entre muchos otros) Por ejemplo, no acepta la substitución total y perfecta entre esas formas de capital. Propone que se debe sustentar la integridad de los ecosistemas, entendida como la estructura y características de los sistemas que sustentan la vida y sus complejas interconexiones. La solución al conflicto entre ambiente y progreso es exógena al modelo por lo que requiere un cambio de paradigma que corrija al modelo (Pérez, Rojas y Ordóñez, 2016). Los elementos principales de esta corriente de pensamiento han sido adoptados y ampliados por la economía ecológica. Se incorporan consideraciones técnicas y políticas en la toma de decisiones.

La sustentabilidad superfuerte, se distancia sustancialmente de la ideología del progreso. La naturaleza es valorada desde múltiples perspectivas, y se utiliza el concepto de Patrimonio Natural que implica otros valores más allá del económico. Además, se incorpora una perspectiva ética en la generación de la sustentabilidad, reconociéndose el valor intrínseco de la Naturaleza más allá de la utilidad potencial para el ser humano. Esta corriente propone nuevos estilos de desarrollo, con cambios profundos en los procesos productivos y en la conceptualización y aplicación de conceptos tradicionales tales como eficiencia, rentabilidad, equidad, etc. También, reconoce una pluralidad de valoraciones en los seres humanos, y por lo tanto la toma de decisiones sobre el desarrollo son esencialmente políticas al requerir la argumentación entre posturas diferentes.

\subsection{Campus Sostenible}

En los últimos años se ha abordado el concepto de Universidad o Campus Sostenible (CS) como una estrategia de gestión. Castañeda \& Quintero (2015) lo definen como aquella institución de educación superior que dirige sus esfuerzos operacionales (administrativos), de investigación, de extensión y docencia (sustantivos) hacia el manejo de los riesgos socioambientales, con el propósito de reconocer que sus impactos modifican el entorno biótico (humano y no humano) y abiótico, y de garantizar el mantenimiento de las condiciones sociales, ecológicas y económicas en sus grupos de interés. En general, las universidades son vistas como instituciones educativas formales, pero las universidades también ofrecen oportunidades de 
aprendizaje informal, dando el ejemplo y proveyendo el espacio para el desarrollo de diversas actividades y relaciones. La consistencia entre el discurso y la acción aporta legitimidad al discurso y promueve el comportamiento ético (Paterson y Huang, 2018). Las acciones educativas no se limitan a la educación formal, sino que se extienden al amplio campo de la educación informal y en línea que favorece la difusión global y una conectividad constante que debe ser aprovechada críticamente (Hayden, 2008). Las actuaciones de los docentes y de la institución en general son un amplificador moral para los estudiantes (Hanson et al., 2017).

\section{FIGURA I. Modelo propuesto}

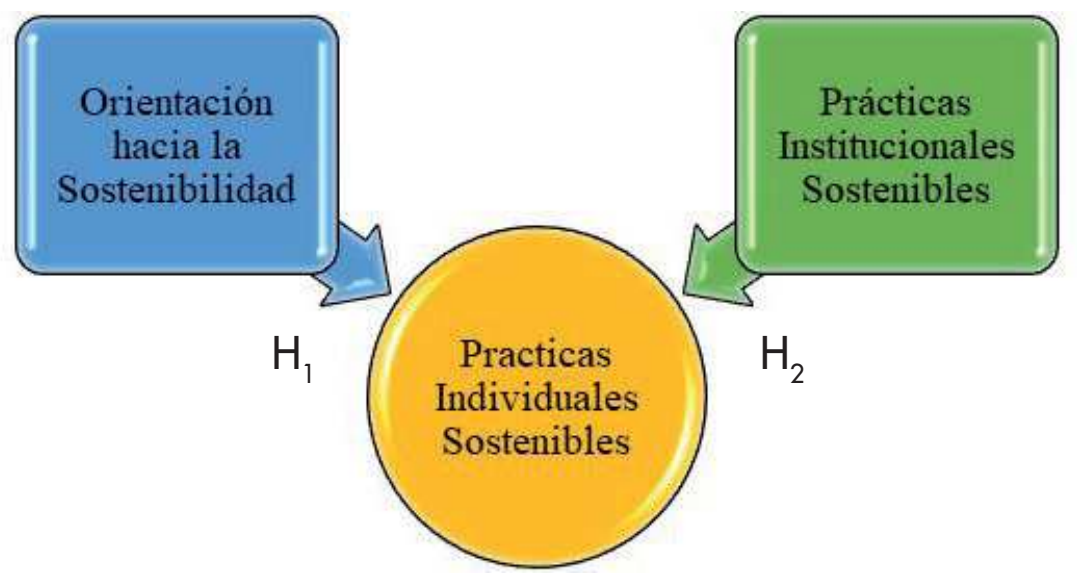

\subsection{Marco teórico}

La Figura 1 muestra el modelo, el cual propone que mientras más fuerte sea la orientación hacia la sostenibilidad y más alta su percepción de prácticas institucionales sostenibles más prácticas sostenibles adoptará el individuo. En esa dirección se examinarán las siguientes hipótesis generales:

$\mathrm{H}_{1}$ : La correlación del Índice de Orientación a la Sostenibilidad estará más positivamente correlacionado al Índice de Practicas Individuales cuanto más fuerte sea la orientación hacia la sostenibilidad.

$\mathrm{H}_{2}$ : El Índice de Campus Sostenible está positivamente correlacionado al Índice de Practicas Individuales.

Además, la adopción de prácticas y hábitos sostenibles se examina a la luz del modelo transteórico de etapas de cambio de Prochaska \& Di Clemente (1984), ya que se trata no solo de un cambio de paradigma, sino también de comporta- 
miento. En esa dirección argumentamos que en la etapa de pre-contemplación los individuos no le dan mucho pensamiento al asunto de la sostenibilidad y es muy probable que no esté inclinado a tomar ninguna acción, lo que en este estudio asociamos a la orientación no sustentable. En la fase de contemplación el individuo está más consciente del asunto en cuestión y comienza a mostrar algunos signos de inquietud y se abre a nueva información, caracterizándose por una actitud reformista, lo cual asociamos a la orientación hacia la sostenibilidad débil. En la etapa de preparación la persona comienza a motivarse y su actitud hacia el cambio se va haciendo más positiva y se va convenciendo de que algo hay que hacer lo cual asociamos a la orientación fuerte. En la etapa de acción la persona cambia el comportamiento basado en una fundamentación profunda que se traduce en la ejecución de pequeñas o grandes acciones sostenibles lo cual asociamos a la orientación superfuerte.

\section{Metodología}

Este estudio es parte de una investigación mayor. Los resultados de la investigación realizada se publicaron en dos documentos. En el primero, (López Paláu y Rivera Cruz, 2020) se presentaron los resultados del análisis descriptivo de los datos proveyendo un primer diagnóstico de la percepción de estudiantes de negocios sobre las practicas sostenibles actuales, individuales e institucionales, y los fundamentos de su orientación hacia la sostenibilidad. En este segundo documento se presentan los resultados del análisis correlacional añadiendo al marco teórico el modelo transteórico de etapas de cambio de Prochaska \& Di Clemente (1984) para su interpretación. Los datos fueron recolectados mediante un cuestionario con tres secciones utilizando escalas bipolares y otra sección de información demográfica. La muestra fue recolectada de forma conveniente.

En la primera sección del cuestionario se les solicitó a los participantes que evaluaran 39 prácticas institucionales sustentables para que ofrecieran su opinión, en términos porcentuales, sobre la frecuencia, amplitud y consistencia con la que se practica cada una en su universidad, utilizando una escala bipolar de $0 \%$ No se Practica hasta 100\% Se Practica. El índice de Campus Sostenible presentado en este segundo documento es el resultado del promedio de las respuestas a las 39 prácticas institucionales.

En la segunda sección del cuestionario se les solicitó a los participantes que evaluarán 43 comportamientos afines con la sustentabilidad que las personas 
pueden adoptar en su vida diaria, para que indiquen, en términos porcentuales, la frecuencia y consistencia con la que practican cada uno, utilizando una escala bipolar de 0\% No lo Practico hasta 100\% Lo Practico. El promedio de las respuestas de esta sección es el índice de Prácticas Individuales.

En la tercera sección del cuestionario se presentaron 22 premisas sobre la sustentabilidad para que los participantes expresaran su acuerdo utilizando una escala bipolar de 0\% Totalmente de Acuerdo hasta 100\% Totalmente en Desacuerdo. Las premisas incluían conceptos y posturas asociadas a las corrientes de sustentabilidad débil, fuerte, superfuerte $o$ insostenibilidad de las cuales se calcularon los cuatro índices mediante el promedio de los elementos correspondientes a cada visión.

\section{I. Métodos estadísticos}

Se realizaron análisis descriptivo, de confiabilidad y correlacional de los índices calculados. También se realizó una regresión jerárquica para examinar las dos hipótesis propuestas. Este método permite que los incrementos en la varianza puedan ser atribuidos a cada serie de variables independientes. De esta forma, el efecto de cada variable añadida es claro. Las pruebas estándares $F$ se utilizaron para determinar la significancia de cada variable a la varianza $Y$. El coeficiente de determinación $R^{2}$ prueba el poder explicativo de la ecuación de regresión, mientras que el $R^{2}$ ajustado permite las comparaciones entre modelos con diferentes variables independientes. Los coeficientes betas estandarizados permiten comparaciones entre ellos, así como el poder explicativo relativo de la variable dependiente.

\section{Resultados}

\section{I. Composición de la muestra}

En la Figura 2 se presentan los detalles de la composición de la muestra. Participaron 168 estudiantes de administración de empresas del recinto principal de la universidad pública de Puerto Rico. La mayor parte de la muestra son mujeres, menores de 25 años, en sus dos últimos años de estudios, trabajan a tiempo parcial, pueden cubrir casi todas sus necesidades o más y viven con sus padres. 


\section{FIGURA 2. Composición de la muestra}

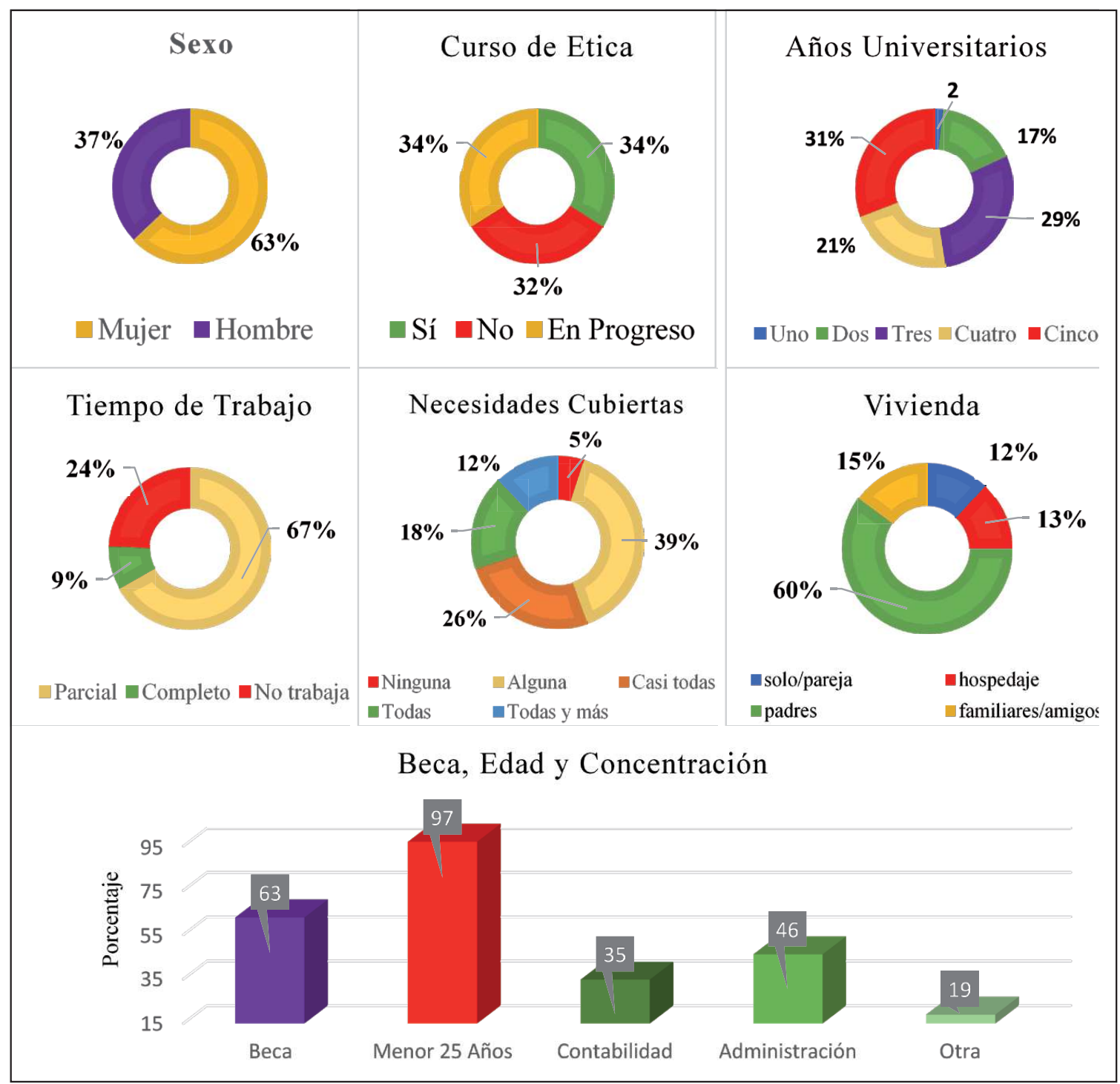

\subsection{Análisis descriptivo y confiabilidad}

En la Tabla 1 se presenta estadísticas descriptivas y los coeficientes Cronbach's Alpha para los índices calculados en este estudio. Los índices se calcularon del promedio de los elementos examinados en cada una de las secciones del cuestionario, las cuales pueden ser revisadas en López Paláu y Rivera Cruz (2020). Dos de los seis índices calculados resultaron en valores sobre .89. Ello refleja una alta confiabilidad y exceden por mucho el límite de .70 generalmente aceptado 
y defendido por Nunally and Berstein (1994) como el mínimo deseable. Los otros cuatro alcanzaron valores entre .34 y .57 , lo cual apunta a la necesidad de revisar los elementos que los definen. Es importante notar que dichos índices se calcularon del promedio de respuestas a cinco elementos por lo que debe considerarse añadir más elementos en su determinación.

Los resultados reflejan que los participantes parecen acercarse más una orientación superfuerte hacia la sustentabilidad que a la postura de la insostenibilidad lo que refleja una cierta toma de conciencia. No obstante, las puntuaciones en los índices débil, fuerte y superfuerte parecen reflejar que los participantes adoptan fundamentos de cada postura reflejando un proceso evolutivo de ampliación y profundización en el tema, cónsono con el modelo transteórico de etapas de cambio de comportamiento en el que se basa el modelo aquí examinado. Además, los resultados revelan que los participantes parecen estar en una etapa de cambio de comportamiento que tímidamente va convirtiéndose en acciones sostenibles, cónsono con las etapas de cambio de preparación y acción, y la percepción de las políticas institucionales es que su universidad está haciendo lo mínimo en ruta a ser considerado un campus sostenible.

\section{TABLA I. Estadísticos descriptivos y confiabilidad de los índices}

\begin{tabular}{|l|c|c|c|}
\hline Índices & Promedio & $\begin{array}{c}\text { Desviación } \\
\text { estándar }\end{array}$ & $\begin{array}{c}\text { Chronbach } \\
\text { Alpha }\end{array}$ \\
\hline Campus Sostenible & 52 & 18 & .95 \\
\hline Prácticas Individuales & 55 & 14 & .89 \\
\hline Orientación No Sustentable & 31 & 17 & .57 \\
\hline Orientación Débil & 53 & 18 & .41 \\
\hline Orientación Fuerte & 71 & 17 & .36 \\
\hline Orientación Super Fuerte & 73 & 16 & .34 \\
\hline
\end{tabular}

En la Tabla 2 se presentan las correlaciones significativas a un nivel de .05 o mayor entre los índices. La correlación más alta es entre la orientación débil y la fuerte reflejando la fundamentación que comparten ambas corrientes seguida por la correlación entre el índice de prácticas individuales y el de campus sostenible proveyendo evidencia de la influencia de las prácticas institucionales en el comportamiento de los estudiantes. Las prácticas institucionales actuales se relacionan con la orientación débil. La correlación de las prácticas individuales aumenta mientras 
más fuerte es la orientación hacia la sostenibilidad según predicho por el marco teórico. La orientación no sustentable se correlaciona positivamente con la orientación débil, demostrando similitud sustancial entre ambas corrientes, mientras que se correlaciona negativamente con la sostenibilidad superfuerte, cónsono también con el marco teórico. Estos resultados proveen validez de contenido, puesto que las relaciones entre las variables se dan conforme a lo previsto por el marco teórico.

\section{TABLA 2. Correlaciones entre los Índices}

\begin{tabular}{|c|c|c|c|c|c|c|}
\hline Índices & 1 & 2 & 3 & 4 & 5 & 6 \\
\hline 1 Campus Sostenible & & .494 & .172 & .253 & & \\
\hline 2 Prácticas Individuales & & & & .166 & .230 & .288 \\
\hline 3 Orientación No Sustentable & & & & .286 & & -.173 \\
\hline 4 Orientación Débil & & & & & .623 & \\
\hline \multicolumn{7}{|l|}{5 Orientación Fuerte } \\
\hline 6 Orientación Super Fuerte & & & & & & \\
\hline
\end{tabular}

(Solo se muestran los coeficientes $\beta$ significativos estadísticamente por lo menos al nivel .05)

\subsection{Resultados de las hipótesis}

Se ejecutó una regresión jerárquica para el Índice de Prácticas Individuales. En el primer paso se entraron los índices de insostenibilidad y orientación débil, fuerte y superfuerte para examinar la primera hipótesis y en el segundo paso se entró a la ecuación el índice de campus sostenible para examinar la segunda. La Tabla 3 presenta los resultados de la regresión.

Primera hipótesis: La correlación del Índice de Orientación a la Sostenibilidad estará más positivamente correlacionado al Índice de Practicas Individuales cuanto más fuerte sea la orientación hacia la sostenibilidad.

Los resultados proveen evidencia de apoyo para esta hipótesis, puesto que sólo la orientación superfuerte tiene un efecto significativo en las prácticas individuales, sugiriendo que los individuos que están más en sintonía con los fundamentos de la sustentabilidad superfuerte adoptan más hábitos sostenibles. La orientación no sustentable, débil o fuerte no se traduce en comportamientos sustentables, como se propone en el modelo. 


\section{TABLA 3. Resultados Regresión de Índice de Prácticas Individuales}

\begin{tabular}{|l|c|c|}
\hline Variables Independientes & Promedio & Desviación estándar \\
\hline Índice Orientación No Sustentable & & \\
\hline Índice Orientación Débil & & \\
\hline Índice Orientación Fuerte & & .198 \\
\hline Índice Orientación Super Fuerte & .270 & .252 \\
\hline Índice Campus Sostenible & & .483 \\
\hline$\Delta \mathrm{R}^{2}$ & .127 & .215 \\
\hline $\mathrm{R}^{2}$ & .127 & .342 \\
\hline $\mathrm{R}^{2}$ Ajustado & .105 & .321 \\
\hline
\end{tabular}

(Solo se muestran los coeficientes $\beta$ significativos estadísticamente por lo menos al nivel .05)

Segunda hipótesis: El Índice de Campus Sostenible está positivamente correlacionado al Índice de Prácticas Individuales.

Los resultados también proveen evidencia de apoyo para esta hipótesis. La percepción de campus sostenible tiene un efecto positivo en las prácticas individuales, según establecido en el modelo propuesto. Aunque el $\mathrm{R}^{2}$ ajustado es modesto, es suficientemente provocador en un estudio exploratorio como este para replicar y ampliar las propuestas del modelo aquí examinado.

\section{Discusión de los Resultados}

En conjunto, los resultados proveen evidencia sobre la importancia de las instituciones de educación superior en la educación de y para la sostenibilidad. También proveen evidencia de apoyo al modelo transteórico de etapas de cambio en la adopción de comportamientos y hábitos sostenibles, el cual debe ser visto como un proceso dinámico y no como un acto único y aislado. Todo parece indicar que las universidades pueden ser parte vital y un escenario idóneo de dicho proceso no sólo mediante la generación y transmisión de conocimiento, sino creando oportunidades de aprendizaje informal a través del ejemplo, adoptando prácticas institucionales sostenibles en todos los ámbitos de su quehacer organizacional. No obstante, pueden ser motor de cambio o lastre, dependiendo de si transmiten o no con su ejemplo y oferta educativa formal e informal, la urgencia de adoptar 
comportamientos sostenibles catalizando o ralentizando el proceso de cambio de sus constituyentes.

\section{Conclusiones}

Este estudio logró los cuatro objetivos propuestos. Primero, se determinaron los índices de orientación hacia sustentabilidad, reflejando que los participantes rechazan la insostenibilidad y se van identificando con los fundamentos más fuertes de la sustentabilidad en un proceso de cambio en etapas. Segundo, se calculó el Índice de Prácticas Individuales Sostenibles encontrando que las practican poco. Tercero, se calculó el índice de Campus Sostenible encontrando que el recinto estudiado también practica poco la sustentabilidad. Cuarto, se determinó el efecto de la orientación hacia la sostenibilidad y la percepción de campus sostenible en los hábitos sostenibles de los estudiantes encontrando evidencia de apoyo para el modelo propuesto, revelando que las instituciones de educación superior pueden ser un magnifico escenario para promover y practicar la sustentabilidad y sus aristas.

En conjunto los resultados revelan que en general los estudiantes se encuentran entre las etapas contemplativa y la preparación. Esta es una etapa media asociada a la orientación débil y fuerte. En esta postura los participantes están abiertos a información y tienen cierta inquietud, pero su acercamiento al tema es todavía superficial, sin profundizar en lo conceptos, las implicaciones y retos que conlleva la sustentabilidad. Aunque se van alejando de las posturas de insostenibilidad. $\backslash$, todavía están en una etapa mas reflexiva. En esta etapa no hay todavía contribución para lograr la transformación que la sustentabilidad requiere. Se encuentran en un proceso de reflexión individual que no les permite aun hacer concertaciones colectivas que promueva un cambio social. Esto implica una responsabilidad mayor para las instituciones de ES que deben promover mayor vinculación con la comunidad a la que sirven en aras de resolver los problemas que le atañen relacionados a la sustentabilidad en su conceptualización más amplia.

\section{Contribución del Estudio}

El modelo aquí propuesto, es meritorio por su valor teórico para avanzar el conocimiento sobre las dimensiones de la sostenibilidad. Primero, el estudio propone un modelo básico en el que por primera vez se considera como variable la orientación hacia la sostenibilidad según las corrientes filosóficas descritas en 
la literatura y la percepción de las prácticas institucionales y su influencia en el comportamiento individual. Segundo, el modelo propuesto integra fundamentos del modelo transteórico de cambio de comportamiento, el cual es una teoría ya madura que ha sido ampliamente validada a través del tiempo, dándole un giro innovador para comprender la adopción del comportamiento sustentable. Tercero, aborda el concepto de la sustentabilidad desde una concepción amplia que incluye aspectos educativos, culturales, éticos, políticos, económicos, etc. Cuarto, define por primera vez los índices de orientación hacia la sustentabilidad. Quinto, tiene implicaciones prácticas importantes, al proveer evidencia de la importancia de los aspectos organizacionales y de política institucional para promover comportamientos sostenibles.

\section{I. Limitaciones}

Dado que la muestra no fue seleccionada aleatoriamente los resultados no han sido generalizados a la población. No obstante, siendo este un estudio exploratorio, la información recopilada tiene un valor incalculable para establecer un entendimiento base sobre la educación de la sostenibilidad y un punto de referencia para estudios posteriores.

\subsection{Palabras finales}

En general, tanto los participantes en su práctica individual como el campus en su práctica institucional están bastante lejos de tener hábitos sostenibles. Este resultado plantea que posiblemente estemos ante un círculo vicioso. En la medida que la institución no se compromete con la sustentabilidad, sus constituyentes no adoptan prácticas sostenibles y en la medida en que los individuos no practican la sustentabilidad es menos probable que reclamen acciones sostenibles y cambios curriculares en la institución. Es importante que las instituciones de educación superior rompan ese círculo y promuevan la sustentabilidad incluyéndola en sus funciones sustantivas y adjetivas, cumpliendo así su responsabilidad social y planetaria.

De igual forma, este estudio es un llamado a docentes e investigadores para que validen el modelo aquí propuesto en sus contextos nacionales. Posibles ampliaciones de esta investigación incluyen el utilizar una muestra de los otros constituyentes universitarios para hacer un diagnóstico más profundo y preciso en aras de identificar vías para romper el círculo vicioso que hemos descrito en este estudio, que ralentiza el proceso hacia la sustentabilidad. 


\section{Referencias}

Alcaniz, L., Aguado, R. y RetolazA, J. L. (2017). "New business models: beyond the shareholder approach", en Academy of Management Proceedings, Vol. 2017, $\mathrm{N}^{\circ}$ 1, 15.492, Briarcliff Manor, NY 10510, Academy of Management.

Barth, M., Godemann, J., Rieckmann, M. y Stoltenberg, U. (2007). "Developing key competencies for sustainable development in higher education", International Journal of Sustainability in Higher Education 8(4), 416-430.

BRUNDTLAND, G. (1987). Report of the World Commission on Environment and Development: Our Common Future. United Nations General Assembly document A.

CABEZA, M. (1996). "The concept of weak sustainability", Ecological Economics 17: 147-156.

Castañeda, Á. M. P., y Quintero, H. F. T. (2015). "Universidad y Sostenibilidad: Una Aproximación Teórica para su Implementación", $\operatorname{Ad-Minister~(26),~149-163.~}$

Costanza, R. y PATten, B. (1995). "Commentary: Defining and predicting sustainability", Ecological Economics 15, 193-196.

CostanZA, R. (1991). Ecological Economics: The Science and Management of Sustainability, Nueva York, Columbia University Press.

CoRTés, F. (2014). "Fundamentos filosóficos de una propuesta de reforma de la educación superior", Co-herencia 10 (20), 215-233. Medellín (ISSN 1794-5887).

Daly, H. y CоOB, C. (1989). For the Common Good, Boston, Beacon Press.

Estrada, O. y Olivares, A. (2017). "Implicaciones del desarrollo territorial en los territorios dependientes: evaluación del caso de Puerto Rico". Bitácora Urbano Territorial 27(3), 29-42.

FreEstone, O. M., y McGoldRICK, P. J. (2008). "Motivations of the ethical consumer", Journal of Business Ethics 79(4), 445-467.

GudYNAS, E. (2004). "Ecología, economía y ética del desarrollo sostenible", Montevideo, Coscoroba, www.ecologiapolitica.net

- (2009). "Desarrollo sostenible: posturas contemporáneas y desafíos en la construcción del espacio urbano", Vivienda Popular 18, 12-19, Montevideo, Facultad de Arquitectura. 
- (201 1). "Desarrolloy sustentabilidad ambiental: diversidad de posturas, tensiones persistentes", en A. MATARÁN y F. López (coords.), La Tierra no es muda: diálogos entre el desarrollo sostenible y el postdesarrollo, 35-52, Granada, Universidad de Granada.

Hall, K. L. y Rossl, J. S. (2008). Prev Med.; 46(3):266-74. doi: 10.1016/j. ypmed.2007.11.006.

Hanson, W. R.; Moore, J. R.: Bachleda, C.; Canterbury, A.; Franco, C.; Marion, A. y SCHREIBER, C. (2017). "Theory of Moral Development of Business Students: Case Studies in Brazil, North America, and Morocco", Academy of Management Learning \& Education 16(3), 393-414.

HAYDEN, T. (2008). El estado del planeta, National Geographic España, Madrid, RBA.

HoWARTH, R. B. y NoRGAARD, R. B. (1993). "Intergenerational transfers and the social discount rate", Environmental and Natural Resources Economics 3(4), 337-358.

Labanauskis, R. (2017). "Key Features of Sustainable Universities: A Literature Review", Journal of Business Management 13. https://tinyurl.com/2p926wbd

Lipschitz, J. M., Yusufov, M., Paiva, A., Redding, C. A., Rossi, J. S., Johnson, S., Blissmer, B., GokbaYrak, N. S., Velicer, W. F. y Prochaska, J. O. J. (2005). Sport Exerc Psychol 37(6), 592-606. doi: 10.1123/jsep.2014-0329.PMID: 26866767

López Paláu y Rivera-Cruz (2018). "Orientación de los Consumidores Mileniales de Puerto Rico hacia el Desarrollo Sostenible y sus Movimientos Afines desde la Perspectiva de la Ética de la Virtud", Asamblea ALAFEC, Nayarit, México.

- (2019). Intenciones Agroemprendedoras de Estudiantes Universitarios de Puerto Rico y su Inquietud Ética hacia el Desarrollo Sostenible. Working Paper

- (2020). Estudio Empírico sobre la Orientación y Hábitos Sostenibles de Estudiantes Universitarios de Puerto Rico y las Prácticas Institucionales en su Campus. Working paper.

Martínez-Alier, J. y RocA, J. (2013). [3ª edición] Economía Ecológica y Política Ambiental, 639 pp, México, FCE.

Mejía, E. y SeRna, C. (2015). "La contabilidad en función de la sustentabilidad: una mirada desde el desarrollo económico alternativo", Quipukamayoc XXIII(44), 109-118. 
Migneault, J. P., Velleer, W. F., Prochaska, J. O. y Stevenson, J. F. (1999). “Decisional balance for immoderate drinking in college students", Subst Use Misuse 34, $1325-46$.

MILER, W. R. y RolLNICK, S. (1991). Motivational interviewing: preparing people to change addictive behavior, Nueva York, Guilford Press.

Nunnally, J. y Berstein, I. H. (1994). [3ª edición] Psychometric Theory, Nueva York, McGraw-Hill.

Paterson, T. A., y Huang, L. (2018). "Am I expected to be ethical? A role-definition perspective of ethical leadership and unethical behavior", Journal of Management, 0149206318771166.

Pérez, M., Rojas, J. y Ordóñez, C. (Comp.) (2016). Desarrollo Sostenible: Principios, enfoques y lineamientos de política para Colombia, Programa Editorial Universidad del Valle.

Piza, V., Aparicio, J. L., Rodríguez, C., Marín, R., Beltrán, J. y Bedolla, R. (2018). "Sustainability in Higher Education: A Didactic Strategy for Environmental Mainstreaming", Sustainability, 10, 4556.

ProchaskA, J. O. y DiClemente, C. C. (1983). "Stages and processes of self-change of smoking, toward an integrative model of change", Journal of Consulting and Clinical Psychology 51, 390-395, doi:10.1037/0022-006X.51.3.390

- (1984). The transtheoretical approach: Crossing traditional boundaries of therapy, Homewood, IL, Dow Jones-Irwin.

Prochaska, J. O., DiClemente, C. C., \& Norcross, J. C. (1992). "In search of how people change: Applications to the addictive behaviors", American Psychologist 47, 1102-1114. PMID: 1329589.

Prochaska, J. O., DiClemente, C. C., Velicer, W. F. y Rossi, J. S. (1993). "Standardized, individualized, interactive, and personalized self-help programs for smoking cessation", Health Psychology 12, 399-405.Google Scholar

Prochaska, J. O., Norcross, J. C. y DiClemente, C. C. (1994). Changing for good: A revolutionary six-stage program for overcoming bad habits and moving your life positively forward, Nueva York, Avon Books. 
Prochaska, J. O. y DiClemente, C. C. (2005). "The transtheoretical approach", en J. C. Norcross y M. R. GoldFried (Eds.), Handbook of psychotherapy integration, 141-171, Oxford University Press, https://doi.org/10.1093/med:psy $\mathrm{ch} / 9780195165791.003 .0007$

Rivera Cisneros, A. (2017). "Exploración de modelos para el cambio personal y social: el modelo transteórico de Prochaska", Revista Oratores (1).

RiverA, B. y López, S. (2017). "Bioethical Reasoning and the Propensity of Millennial Consumers to Adopt SustainableDevelopmentBehaviors" (Ed), Research in Innovation and Entrepreneurship Series (Johns Hopkins University), Edward Elgar Publishing.

Rossl, M. S., Brown, H. S. y BASS, L. W. (2000). "Leaders in sustainable development: how agents of change define the agenda", Business Strategy and the Environment 9, 273-286.

SANDRI, O., HOLDSWORTH, S. y THOMAS, I. (2018). "Vignette question design for the assessment of graduate sustainability learning outcomes", Environmental Education Research 24(3), 406-426.

Sото, G. (2012). "Desarrollo sustentable o ética ambiental", Artículos y Ensayos de Sociología Rural 7 (13), 7-19.

VICTOR, P. (1991). "Indicators of sustainable development: some lessons from capital theory", Ecological Economics 4, 191-213.

VILA BIAGGI, I. (2016). "El país desfigurado: Una mirada a los problemas estructurales que impiden el progreso congruente y sustentable de Puerto Rico", Revista Jurídica 85, 769, Universidad de Puerto Rico.

VíLCHEZ, A. y GIL, D. (2012). "La educación para la sostenibilidad en la universidad: el reto de la formación del profesorado", Profesorado 16 (2), 25-43. hitp://www. ugr.es/ recfpro/

Vílchez, A., GiL, D., Toscano, L. C. y Macías, O. (2020). Ciencia de la Sostenibilidad, http://www.oei.es/decada/accion.php?accion=24

VILELA, M. (2014). "Conferencia Magistral Valores de la Sustentabilidad, Educación y Carta de la Tierra", Cátedra UNESCO de Educación para la Paz Universidad de Puerto Rico, https://tinyurl.com/yckbpk7c 